\title{
Follow-Up Management of Patients With Testicular Cancer: A Multidisciplinary Consensus-Based Approach
}

\author{
Clair J. Beard, MDª; Shilpa Gupta, MD ; Robert J. Motzer, MDc; Elizabeth K. O’Donnell, MDd; \\ Elizabeth R. Plimack, MD, MS ${ }^{\text {e }}$ Kim A. Margolin, MDf; Charles J. Ryan, MD; ; Joel Sheinfeld, MD ${ }^{\text {h }}$; and \\ Darren R. Feldman, MDc
}

\begin{abstract}
Testicular cancer is the most common cancer in men aged 15 to 40 years in the United States, Canada, and many European countries. Given the excellent prognosis of most men with testicular cancer, updates in care after treatment have become very important. This article provides a review of the available evidence, integrated with expert medical judgment, in the area of testicular cancer followup. (J Natl Compr Canc Netw 2015;13:811-822)
\end{abstract}

Testicular cancer is the most common cancer in men aged 15 to 40 years in the United States, Canada, and many European countries. ${ }^{1,2}$ The incidence of testicular cancer is increasing in all of these areas, and in several countries it has doubled in the past 20 years. ${ }^{3}$ However, available evidence suggests that death from testicular cancer is now rare $(<1 \%-5 \%)$ in appropriately managed patients in the United States, Canada, and Europe. ${ }^{4,5}$

\footnotetext{
From aDepartment of Radiation Oncology, Dana-Farber Cancer Institute/Brigham and Women's Hospital, Boston, Massachusetts; bivision of Medical Oncology, Moffitt Cancer Center, Tampa, Florida; 'Department of Medicine, Memorial Sloan Kettering Cancer Center, New York, New York; dDivision of Medical Oncology, Dana-Farber Cancer Institute/Brigham and Women's Hospital, Boston, Massachusetts; 'Department of Hematology/Oncology, Fox Chase Cancer Center, Philadelphia, Pennsylvania; ${ }^{f}$ Division of Oncology, Stanford University Medical Center, Palo Alto, California; 'Division of Hematology/Oncology, The University of California, San Francisco, California; hUrology Service, Department of Surgery, Memorial Sloan Kettering Cancer Center, New York, New York. Submitted January 28, 2015; accepted for publication May 18, 2015.

Dr. Feldman has disclosed that he is a consultant and/or advisory role for Gilead, research support from Bayer, and consultant for Seattle Genetics. The remaining authors have disclosed that they have no financial interests, arrangements, affiliations, or commercial interests with the manufacturers of any products discussed in this article or their competitors.

Portions of this manuscript were published previously in the 2015 Genitourinary Cancers Symposium Daily News. Sweeney C, Beard C. Approaches to Managing Clinical Stage I Testicular Cancer. Available at: http://gucasym.org/approaches-managing-clinicalstage-i-testicular-cancer. Accessed June 8, 2015.

Correspondence: Clair J. Beard, MD, Dana-Farber Cancer Institute/ Brigham and Women's Hospital, 75 Francis Street, ASB-1, Level 2, Boston, MA 02115. E-mail: cbeard@Iroc.harvard.edu
}

Given the excellent prognosis of most men with testicular cancer, updates in care after treatment have become very important. After the diagnosis and treatment of testicular cancer, the goal of follow-up care is to identify relapse early enough that effective salvage therapy can be given. Over time, as the risk of relapse diminishes, the focus of care changes to identifying and treating the late effects that patients with testicular cancer are known to experience. This article focuses on the follow-up care given in the first 5 years after the diagnosis and treatment of testicular cancer.

\section{Follow-Up for Testicular Cancer}

Follow-up care for patients with testicular cancer begins immediately after orchiectomy for those with clinical stage I disease, defined as no evidence of cancer beyond the testicle, who choose an active surveillance management strategy. Active surveillance implies careful monitoring of patients, with treatment reserved exclusively for those who experience disease relapse. Patients with stage I disease must have no postorchiectomy tumor marker elevation and no evidence of cancer in any lymph node chain or soft tissue. ${ }^{6}$ Beyond a good history and physical examination, the standard baseline workup for men with testicular cancer consists of obtaining preorchiectomy tumor markers, specifically $\alpha$-fetoprotein (AFP), $\beta$-human chorionic gonadotropin $(\beta-\mathrm{HCG})$, and lactate dehydrogenase $(\mathrm{LDH})$, and postorchiectomy markers markers with the exception of patients with clinical stage I seminoma in whom postorchiectomy markers may not be helpful in the detection of recurrent cancer. ${ }^{7}$ An abdominopelvic CT scan is the universally accepted imaging technique for evaluating both lymph nodes and abdominal soft tissues. The lymph nodes most at risk of involvement by testicular 
cancer are those in the retroperitoneum: the paraaortic and aortocaval chains. Lymph nodes are considered positive when the short axis of the node, as measured on an axial CT slice, is $10 \mathrm{~mm}$ or more. Alternatives to CT scans, such as ultrasonography of the retroperitoneum and MRI, are generally not used. Imaging of the chest is mandatory and although a chest radiograph is sufficient for those with stage I seminoma, a chest CT is mandatory for any patient with nonseminomatous cancer elements or for those with stage II or higher seminoma only at diagnosis. In the NCCN Clinical Practice Guidelines in Oncology (NCCN Guidelines) for Testicular Cancer, ${ }^{8}$ a chest radiograph is currently the preferred imaging modality for follow-up after primary therapy and for surveillance in patients with clinical stage I disease, provided that patients are asymptomatic. The American Joint Committee on Cancer (AJCC) stages for testicular cancer and The International Germ Cell Cancer Collaborative Group (IGCCCG) classification of risk groups for metastatic disease are available online, in the NCCN Guidelines for Testicular Cancer, available at NCCN.org (ST-1).

Several considerations are involved in developing a follow-up regimen. Excessive follow-up is both costly and potentially dangerous, given that both CT scans and radiographs expose patients to ionizing radiation which accumulates with each exposure. Tumor marker blood draws are expensive and occasionally render false-positives, which can lead to unnecessary concern and imaging. Finally, patient compliance with follow-up is an important and poorly studied consideration. In one study based on data from insurance companies in California, community patients received far fewer follow-up interventions than called for by the national guidelines of the study period. ${ }^{9}$ However, in a Canadian study of active surveillance strategies in 6 different centers, compliance with follow-up was best for those protocols that were the least intensive. ${ }^{10}$

Guidelines for testicular cancer are generally broadly divided into 2 groups: those for seminomatous germ cell tumors (GCTs) and those for nonseminomatous GCT (NSGCTs), because these have very different natural histories and risks of recurrence. Follow-up guidelines for both seminomatous and NSGCT are further divided by stage and tumor marker status, because these parameters correlate closely with outcomes after initial therapy.

\section{Nonseminomatous Testicular Germ Cell Tumors}

\section{Low-Risk Clinical Stage I NSGCT (IA)}

Low-risk NSGCTs are defined as those with T1 disease (ie, no lymphovascular invasion). Without lymphovascular invasion, the risk of recurrence after orchiectomy alone is approximately $15 \%$ to $20 \%{ }^{11-14}$ The Swedish-Norwegian Testicular Cancer Study Group reported data on 745 patients with clinical stage I NSGCT treated in the community according to uniform guidelines using active surveillance or chemotherapy for low-risk patients and chemotherapy for high-risk patients. ${ }^{15}$ The results showed that patients with low-risk disease who underwent surveillance had relapse rates of only $11.5 \% .{ }^{12}$ Given that most patients will not experience disease relapse in the absence of additional therapy, active surveil lance is a popular treatment strategy. ${ }^{6,16,17}$ However, no consensus exists regarding the optimal surveillance schedule.

A recently published prospective trial of 414 patients with clinical stage I NSGCT randomized patients between abdominal CT scans at 3 and 12 months (2 scans) versus CT scans at 3, 6, 9, 12, and 24 months (5 scans) after orchiectomy. ${ }^{18}$ The study was $90 \%$ powered to detect a $3 \%$ difference in the number of patients experiencing relapse with intermediate- or poor-risk disease as defined by the IGCCCG. All patients were seen in clinic monthly for the first year, every 2 months for the second year, every 3 months for the third year, and every 4 to 6 months thereafter for tumor markers and a chest radiograph. Relapse rates for the 2 arms were $15 \%$ and $20 \%$, respectively $(P=.21)$, with no difference in the risk of relapse with either intermediate- or poor-risk disease and with no difference in salvage regimens at relapse. At 40 months' median follow-up, overall survival was $100 \%$. Patient compliance was excellent ( $\geq 85 \%$ ) for both study arms. In the 2-scan arm, the first indication of relapse was slightly more likely to be seen through tumor markers compared with the 5 -scan arm (43\% vs 35\%), but the difference was not statistically significant because of the small number of relapses $(P=.08)$. All patients who experienced relapse in this study did so by 24 months, with most relapses occurring by 12 months. Notably, only $10 \%$ of the study patients had lymphovascular invasion; the study authors therefore indicated that the results 
were best applied to low-risk patients. The study included no patients with embryonal-predominant disease.

This study ruled out with $90 \%$ probability an increase in the proportion of patients relapsing with intermediate- or poor-risk disease of more than $1.6 \%$ if they had 2 instead of 5 CT scans as part of surveillance. ${ }^{18}$ The implications of this study are that only 2 CT scans during the first 12 months are necessary, especially for low-risk patients, provided that patients comply with the schedule of frequent office visits, tumor markers, and chest radiographs for up to 24 months. The study authors did not believe that chest CTs were a necessary component of surveillance for low-risk patients. This study did not address the issue of whether ongoing office visits, tumor markers, or imaging were necessary beyond 24 months. Other data relevant to this patient population suggest that occasional relapses occur beyond 24 months. Various studies of surveillance strategy have demonstrated that approximately $80 \%$ of relapses occur during the first 12 months of follow-up, $9 \%$ to $12 \%$ during the second year, and $6 \%$ to $8 \%$ during the third year, further decreasing to $1 \%$ during subsequent years. ${ }^{19-21}$ Inspection of guidelines from a Swiss, German and Austrian multidisciplinary group, ${ }^{22,23}$ from the European Society of Clinical Oncology (ESMO), ${ }^{24}$ and from a group of British testicular cancer specialists ${ }^{25}$ reveals that all recommend ongoing follow-up beyond 2 years.

Given the absence of prospective data on follow-up schedules beyond 2 years, the author's recommendation is to continue follow-up but with a less intensive schedule. NCCN Guidelines include recommendations for active surveillance for clinical stage IA NSGCTs (Table 1). ${ }^{8}$

\section{High-Risk Clinical Stage I NSGCT (IB)}

Multiple studies have identified risk factors for relapse for clinical stage I NSGCT, with presence of lymphovascular invasion being the most significant predictor. ${ }^{13,26,27}$ High-risk clinical stage I NSGCT is defined as a tumor with any lymphovascular invasion. 14,26,27 These tumors have a different natural history than low-risk tumors and are more likely to recur. Numerous studies have shown that recurrence after orchiectomy for these tumors is around $40 \%$ to $60 \% .^{12,28-31}$ Management strategies for these patients include active surveillance, with treatment reserved exclusively for those who experience relapse, 1 to 2 cycles of bleomycin/etoposide/cisplatin (BEP) chemotherapy, or retroperitoneal lymph node dissection (RPLND), although RPLND is reserved for specialty centers and is currently less often used. ${ }^{6}$ All 3 strategies are well studied, and adjuvant BEP chemotherapy versus RPLND was subjected to a prospective randomized study by Albers et al. ${ }^{31}$ Published consensus-based guidelines and data on the follow-up guidelines were used by the investigators reporting the outcomes. Finally, given the effectiveness of cisplatin-based salvage therapy for those who experience relapse, the survival rate is similar for all 3 strategies, and approaches 99\%.,31

Active Surveillance: The higher risk of relapse after orchiectomy for these cancers does not rule out active surveillance as an appropriate management strategy, although most follow-up protocols are more intensive than those used for low-risk tumors. The strategy of using active surveillance for all clinical stage I NSGCT is referred to as "non-risk-adapted active surveillance," because high-risk patients are not separated from low-risk patients and are not

Table 1 Clinical Stage IA, NSGCT: Active Surveillance

\begin{tabular}{|llllll|} 
& \multicolumn{5}{c|}{ Year (at month intervals) } \\
\cline { 2 - 6 } & 1 & 2 & 3 & 4 & 5 \\
\hline $\begin{array}{l}\text { H\&P and } \\
\text { markers }\end{array}$ & Every 2 mo & Every 3 mo & Every 4-6 mo & Every 6 mo & Annually \\
$\begin{array}{l}\text { Abdominal/ } \\
\text { pelvic CT }\end{array}$ & Every 4-6 mo & Every 6-12 mo & Annually & - & - \\
$\begin{array}{l}\text { Chest x-ray } \\
\text { A }\end{array}$ & At mo 4 and12 & Annually & Annually & Annually & Annually \\
\hline
\end{tabular}

\footnotetext{
${ }_{b}^{\text {T}}$ Testicular ultrasound for any equivocal exam.

Chest x-ray may be used for routine follow-up, but chest CT is preferred in the presence of thoracic symptoms.

From the NCCN Clinical Practice Guidelines in Oncology: Testicular Cancer, Version 2, 2015. Available at: NCCN.org. Accessed May 28, 2015; with permission. To view the most recent version of these guidelines, visit NCCN.org.
} 
treated with adjuvant therapies. ${ }^{16,32}$ Investigators at Princess Margaret Hospital followed 125 high-risk patients with the following protocol: tumor markers and chest radiograph every 2 months for 2 years, every 4 months in year 3, every 6 months in year 4 , and annually in year 5 , along with an abdominopelvic CT scan every 4 months for the first 2 years. ${ }^{16}$ Results from this trial were presented collectively with those of 240 patients with low-risk stage I NSGCT followed at the same institution. Of 371 patents, 28\% experienced relapse. The median time for relapse for the entire group was 7.1 months, but $3.8 \%$ of those who experienced relapse did so beyond 2 years. ${ }^{16}$ Of interest, 3 of 4 patients with disease relapses beyond 24 months did so after 5 years. The retroperitoneum was the first site of relapse in most patients (75\%), followed by isolated tumor marker elevation (10\%) and recurrence in the lungs (9\%). Abdominopelvic CT scans (taken every 4 months) and tumor markers (obtained every 2 months) were the most useful components of the follow-up strategy. Although chest radiographs were positive at relapse $16.3 \%$ of the time, they were never positive in isolation.

Similar results were obtained in the Pacific Northwest using similar follow-up schedules. ${ }^{32}$ A total of 233 patients with clinical stage I NSGCT referred to the British Columbia Cancer Agency and the Oregon Testis Cancer Program from 1998 to 2007 were retrospectively reviewed, with 223 patients being on active surveillance. Vascular invasion was present in $27 \%$ of patients, status was unknown in $7 \%$, and embryonal predominance was present in 49\%; overall, approximately $30 \%$ of patients had high-risk disease. The patients were followed according to the following active surveillance protocol. At British Columbia
Cancer Agency, tumor markers and clinical examination were performed every 2 months for 2 years, every 3 months for years 3 through 5 , then annually for another 5 years; chest radiographs and abdominopelvic CT scans were performed every 3 months for the first 2 years, then every 6 months for years 3 through 5, and then annually for another 5 years. At the Oregon Testis Cancer Program, tumor markers, clinical examination, and chest radiographs were performed every 6 weeks for the first year, every 2 months for the second year, every 6 months for years 3 through 5, and then annually for another 5 years. Abdominopelvic CT scans were only performed during the first 2 years: every 3 months for the first year, then every 4 months for the second year. Relapses were seen in 59 patients (26\%), with $85 \%$ of relapses occurring within the first year, $88 \%$ within 2 years, and only $3 \%$ beyond 2 years. Notably, 30 of 59 patients with relapses (51\%) had lymphovascular invasion and all but 1 patient experienced relapse with IGCCCG good-risk disease. ${ }^{5}$ Disease-specific survival at a median follow-up of 52 months was 100\%. In this large multi-institutional study reporting on 1139 patients with clinical stage I NSGCT, relapses were detected first by CT scans in $41 \%$ to $48 \%$ of those who experienced relapse, and by tumor markers in $38 \%$ to $61 \%$, depending on their risk category. ${ }^{5}$ These studies helped inform the development of guidelines for surveillance. The NCCN Guidelines for risk-adapted surveillance in patients with highrisk clinical stage I are presented in Table $2 .{ }^{8}$ The authors continue to recommend chest radiographs as part of the follow-up strategy, but also chest CT scans in patients with thoracic symptoms. However, the surveillance strategy for these high-risk versus

\section{Table 2 Clinical Stage IB, NSGCT: Active Surveillance}

\begin{tabular}{|llllll|} 
& \multicolumn{5}{c|}{ Year (at month intervals) } \\
\cline { 2 - 6 } & $\mathbf{1}$ & $\mathbf{2}$ & $\mathbf{3}$ & $\mathbf{4}$ & $\mathbf{5}$ \\
\hline $\begin{array}{l}\text { H\&P and } \\
\text { markers }\end{array}$ & Every 2 mo & Every 3 mo & Every 4-6 mo & Every 6 mo & Annually \\
$\begin{array}{l}\text { Abdominal/ } \\
\text { pelvic CT }\end{array}$ & Every 4 mo & Every 4-6 mo & Every 6 mo & Annually & - \\
Chest $x$-ray $^{\text {b }}$ & Every 2 mo & Every 3 mo & Every 4-6 mo & Every 6 mo & Annually \\
\hline
\end{tabular}

aTesticular ultrasound for any equivocal exam.

${ }^{b}$ Chest x-ray may be used for routine follow-up, but chest CT is preferred in the presence of thoracic symptoms.

From the NCCN Clinical Practice Guidelines in Oncology: Testicular Cancer, Version 2, 2015. Available at: NCCN.org. Accessed May 28, 2015; with permission. To view the most recent version of these guidelines, visit NCCN.org. 
low-risk stage I NSGCTs differ in the number of visits and CT scans (8-9 vs 4-6) over the 5-year period after diagnosis.

Adjuvant Therapy: Historically, high-risk tumors were treated with RPLND. With an experienced surgeon, relapses in the retroperitoneum after RPLND are rare and survival rates are high, albeit at the risk of complications, such as infertility. Improvements in RPLND techniques over time eliminated most surgical morbidity, ${ }^{33,34}$ but the $10 \%$ to $30 \%$ of highrisk tumors that relapsed did so outside of the retroperitoneum and required chemotherapy salvage. In Europe, cisplatin-based chemotherapy, usually BEP, became popular, and 2 cycles of BEP decreased the relapse rate in high-risk patients to $0 \%$ to $5 \% .{ }^{35} \mathrm{Re}$ sults of a prospective trial in Germany suggest that RPLND should be performed only in Centers of Excellence for this surgery. ${ }^{31}$ In Centers of Excellence, the risk of failure after RPLND more approximates the results seen with BEP. ${ }^{31}$ NCCN Guidelines for follow-up after 1 cycle of BEP chemotherapy reflect the extremely low risk of recurrence with this treatment and are shown in Table 3.8

\section{Clinical Stage II-III NSGCT}

Chemotherapy With or Without RPLND: The impact of treatment selection on relapse-free and disease-specific survival in 242 patients with clinical stage IIA/B disease was evaluated in a nonrandomized comparison at a single institution; 136 patients underwent RPLND and 116 patients underwent primary chemotherapy with 4 cycles of etoposide and cisplatin (EP) followed by RPLND. ${ }^{36}$ In this study, chemotherapy was offered to patients with elevated postorchiectomy markers, retroperitoneal adenopa- thy greater than $2 \mathrm{~cm}$, adenopathy located outside the primary landing zone, and the presence of multiple enlarged lymph nodes. Primary RPLND was the recommended treatment modality for patients with normal postorchiectomy serum tumor markers and a solitary retroperitoneal mass smaller than $2 \mathrm{~cm}$ limited to the primary landing zone. Postchemotherapy RPLND was recommended for patients with residual masses of any size and was performed in most patients. Chemotherapy followed by RPLND was associated with a significantly improved 5-year relapse-free survival (98\% vs 79\%; $P<.001$ ) compared with RPLND alone, but the 5-year disease-specific survival did not differ significantly $(100 \%$ vs $98 \% ; P=.3)$ because of the excellent salvage with cisplatin-based chemotherapy regimens. By offering upfront chemotherapy and RPLND to patients with the highest-risk stage IIA and IIB disease and reserving RPLND for the others, $51 \%$ of those treated with RPLND were able to avoid chemotherapy altogether, and those who received chemotherapy received only 2 cycles. The Indiana University experience with clinical stage II NSGCT using primary RPLND demonstrated that $23 \%$ of patients with clinical stage II disease had pathologic stage I disease, and the relapse rate was $5 \%$ in this group. ${ }^{37}$ Abdominal imaging (with the exception of a new postoperative baseline 3-4 months postsurgery) is generally not necessary when RPLND is performed at a Center of Excellence, and may be omitted entirely or performed less often.

Rabbani et $\mathrm{al}^{38}$ reported the outcomes of 54 patients with stage II NSGCT with low-volume nodal metastases (pN1) after RPLND. Of 54 patients, 50 were observed without further adjuvant therapy. Of the

\begin{tabular}{|c|c|c|c|c|c|}
\hline & \multicolumn{5}{|c|}{ Year (at month intervals) } \\
\hline & 1 & 2 & 3 & 4 & 5 \\
\hline $\begin{array}{l}\mathrm{H} \& \mathrm{P} \text { and } \\
\text { markers }^{\mathrm{a}}\end{array}$ & Every $3 \mathrm{mo}$ & Every $3 \mathrm{mo}$ & Every $6 \mathrm{mo}$ & Every $6 \mathrm{mo}$ & Annually \\
\hline $\begin{array}{l}\text { Abdominal/ } \\
\text { pelvic } \mathrm{CT}\end{array}$ & Annually & Annually & - & - & - \\
\hline Chest x-ray ${ }^{b}$ & Every 6-12 mo & Annually & - & - & - \\
\hline
\end{tabular}

aTesticular ultrasound for any equivocal exam.

${ }^{b}$ Chest x-ray may be used for routine follow-up, but chest CT is preferred in the presence of thoracic symptoms.

From the NCCN Clinical Practice Guidelines in Oncology: Testicular Cancer, Version 2, 2015. Available at: NCCN.org. Accessed May 28, 2015; with permission. To view the most recent version of these guidelines, visit NCCN.org. 
patients managed expectantly, 22\% experienced relapse with a median follow-up of 1.8 months. On multivariable analysis, only persistent postorchiectomy tumor marker elevation was a significant independent predictor of relapse (relative risk [RR], 8.0; 95\% CI, 2.3-27.8; $P=.001$ ), with $80 \%$ of patients experiencing a relapse compared with $15.6 \%$ of those with normal postorchiectomy markers. In this study, tumor markers were the most common means of identifying relapse. Almost all relapses occurred within 18 months. Another randomized trial demonstrated equivalence between immediate adjuvant cisplatin-based chemotherapy and surveillance for patients undergoing orchiectomy and RPLND with pathologic stage II NSGCT. ${ }^{39}$ Patients who experienced relapse while undergoing surveillance were treated with 3 to 4 cycles of chemotherapy. With a median follow-up of 4 years, a significant decrease was seen in the rate of relapse in patients treated with adjuvant chemotherapy ( $6 \%$ vs $49 \% ; P<.001)$. However, the survival rates were similar in both arms because of excellent salvage with cisplatin-based chemotherapy. All relapses occurred within 24 months, with a median follow-up of 4 years.

For clinical stage IIA NSGCTs without tumor marker elevation, the NCCN Guidelines recommend either primary RPLND or surveillance with follow-up every 6 weeks. ${ }^{8}$ In patients who undergo primary RPLND and are found to have pathologic stage I disease, further surveillance similar to that for low-risk stage I NSGCT is recommended ${ }^{8,40,41}$ (see Table 1). Adjuvant chemotherapy is preferred for advanced pathologic nodal disease, for which the risk of recurrence is $50 \%$ or higher. ${ }^{36,39,42}$ For pathologic $\mathrm{N} 2$ disease, 2 cycles of EP or BEP are recommended, ${ }^{43}$ whereas for pathologic $\mathrm{N} 3$ disease, 4 cycles of EP or 3 cycles of BEP are recommended. ${ }^{44}$ The NCCN Guidelines for follow-up of clinical stage II-III NSGCTs are presented in Tables 4, 5, and 6, divided by both treatment strategy and response to treatment. ${ }^{8}$

\section{Clinical Stage IIA NSGCT With Elevated Tumor Markers}

Approximately $75 \%$ of patients with clinical stage IIA NSGCT will achieve complete response with primary chemotherapy. ${ }^{40,45}$ The European Germ Cell Cancer Consensus Group (EGCCCG), ${ }^{46}$ Indiana University ${ }^{47}$ and $\mathrm{ESMO}^{24}$ guidelines do not recommend RPLND for patients who achieve remission with a residual mass less than $1 \mathrm{~cm}$. The authors recommend surveillance. The long-term experience reported by the Indiana group showed that with a median follow-up of 15.5 years, only $9 \%$ of patients experienced recurrence at a median of 11 months, with half of these recurrences occurring in the retroperitoneum. ${ }^{48}$ Frequent clinical follow-up is recommended, with abdominal imaging currently recommended at least twice during the first 2 years and then annually or as clinically indicated.

For partial responders with the presence of a residual mass greater than $1 \mathrm{~cm}$ and normalized tumor markers, the ESMO guidelines ${ }^{24}$ and others recommend surgical resection of residual masses. Subsequently, patients can be put on surveillance in the presence of teratoma or necrosis (Table 4), ${ }^{8}$ or offered 2 cycles of adjuvant chemotherapy in cases of viable tumor.

\section{Advanced/Metastatic NSGCT}

It is difficult to accurately predict the probability of persistent teratoma or viable tumor in patients with

\begin{tabular}{|c|c|c|c|c|c|}
\hline \multicolumn{6}{|c|}{ Year (at month intervals) } \\
\hline & 1 & 2 & 3 & 4 & 5 \\
\hline $\mathrm{H} \& \mathrm{P}$ and markera & Every $2 \mathrm{mo}$ & Every $3 \mathrm{mo}$ & Every $6 \mathrm{mo}$ & Every $6 \mathrm{mo}$ & Every $6 \mathrm{mo}$ \\
\hline $\begin{array}{l}\text { Abdominal/pelvic } \\
\mathrm{CT}^{\mathrm{b}}\end{array}$ & Every $6 \mathrm{mo}$ & Annually & - & - & - \\
\hline Chest x-rayc,d & Every 6 mo & Every $6 \mathrm{mo}$ & Annually & Annuallye & - \\
\hline
\end{tabular}

aTesticular ultrasound for any equivocal exam.

bPatients who undergo RPLND and are found to have pN0 disease (no tumor or teratoma) need only 1 CT scan at postoperative month 4. ${ }^{\circ}$ Chest x-ray may be used for routine follow-up, but chest CT is preferred in the presence of thoracic symptoms.

${ }^{\mathrm{d}}$ Chest CT if supradiaphragmatic disease at baseline.

${ }^{e}$ Chest $x$-ray is optional at month 36 and 48.

From the NCCN Clinical Practice Guidelines in Oncology: Testicular Cancer, Version 2, 2015. Available at: NCCN.org. Accessed May 28, 2015; with permission. To view the most recent version of these guidelines, visit NCCN.org. 
Follow-Up of Patients With Testicular Cancer

\begin{tabular}{|c|c|c|c|c|c|}
\hline & \multicolumn{5}{|c|}{ Year (at month intervals) } \\
\hline & 1 & 2 & 3 & 4 & 5 \\
\hline $\begin{array}{l}\mathrm{H} \& \mathrm{P} \text { and } \\
\text { markers }^{\mathrm{b}}\end{array}$ & Every $2 \mathrm{mo}$ & Every $3 \mathrm{mo}$ & Every $4 \mathrm{mo}$ & Every 6 mo & Annually \\
\hline $\begin{array}{l}\text { Abdominal/ } \\
\text { pelvic } \mathrm{CT}\end{array}$ & At 3-4 moc & \multicolumn{3}{|c|}{ As clinically indicated } & \\
\hline Chest x-ray ${ }^{d}$ & Every 2-4 mo & Every $3-6$ mo & Annually & Annually & Annually \\
\hline
\end{tabular}

\footnotetext{
aPatients with clinical stage II-A/II-B nonseminoma who undergo primary RPLND and are found to have pN0 disease (no tumor or teratoma, pathologic Stage I) should revert to the surveillance schedule for low-risk NSGCT with the exception that only 1 CT scan is needed postoperatively around month 4 (Table 2).

${ }^{\mathrm{b}}$ Testicular ultrasound for any equivocal exam.

${ }^{\mathrm{c}}$ This schedule assumes a complete resection has taken place.

${ }^{d}$ Chest x-ray may be used for routine follow-up but chest CT is preferred in the presence of thoracic symptoms From the NCCN Clinical Practice Guidelines in Oncology: Testicular Cancer, Version 2, 2015. Available at: NCCN.org. Accessed May 28, 2015; with permission. To view the most recent version of these guidelines, visit NCCN.org.
}

advanced metastatic clinical stage III NSGCT with normalized tumor markers and normal CT scans after completion of chemotherapy. ${ }^{49,50}$ The authors recommend that patients who achieve a complete response on imaging and have normalization of tumor markers after completion of chemotherapy be followed by surveillance or RPLND. No reliable criteria exist to distinguish tumor or teratoma from necrotic debris, and the rate of false-negative CT scans is approximately $20 \% .^{51}$ If a residual mass is present on CT scan, but markers have normalized, resection of the residual disease is recommended. ${ }^{52,53}$ If the residual mass demonstrates necrosis or teratoma, surveillance is recommended, whereas in the presence of viable tumor, 2 cycles of consolidative chemotherapy are indicated. Most relapses in advanced metastatic NSGCT occur during the first 2 years after completion of treatment; the incidence of late relapse is $2 \%$ to $6 \%$, with most occurring more than 5 years after initial treatment. Surgical treatment for localized disease can improve survival. ${ }^{54-56}$ A multidisciplinary approach to metastatic NSGCT has resulted in survival rates of more than $80 \%$. A standard surveillance protocol is recommended after patients experience a complete response. Recommendations for follow-up with physical examinations, tumor marker assessments, chest radiographs, and abdominal imaging come from multi-institutional chemotherapy trials. However, the optimal surveillance protocol has not been determined. The additional utility of chest radiographs in patients with initially marker-positive advanced metastatic NSGCT who experience complete remission with chemotherapy is not established. ${ }^{57}$

Although salvage standard chemotherapy for relapsed/refractory disease can result in long-term clinical remissions in up to $40 \%$ of patients, ${ }^{58}$ complete

\begin{tabular}{|c|c|c|c|c|c|}
\hline & \multicolumn{5}{|c|}{ Year (at month intervals) } \\
\hline & 1 & 2 & 3 & 4 & 5 \\
\hline $\begin{array}{l}\mathrm{H} \& \mathrm{P} \text { and } \\
\text { markers }^{\mathrm{a}}\end{array}$ & Every $6 \mathrm{mo}$ & Every $6 \mathrm{mo}$ & Annually & Annually & Annually \\
\hline $\begin{array}{l}\text { Abdominal/ } \\
\text { pelvic } \mathrm{CT}\end{array}$ & After RPLND & \multicolumn{4}{|c|}{ As clinically indicated } \\
\hline Chest $x$-ray ${ }^{b}$ & Every 6 mo & Annually & Annually & Annually & Annually \\
\hline
\end{tabular}

Testicular ultrasound for any equivocal exam.

${ }^{b}$ Chest x-ray may be used for routine follow-up, but chest CT is preferred in the presence of thoracic symptoms.

From the NCCN Clinical Practice Guidelines in Oncology: Testicular Cancer, Version 2, 2015. Available at: NCCN.org. Accessed May 28, 2015; with permission. To view the most recent version of these guidelines, visit NCCN.org. 
responses are seen less frequently for patients with intermediate- and poor-risk metastatic NSGCT, and 4 cycles of BEP remain the standard of care. Several prognostic models have been developed to guide subsequent therapies. ${ }^{59-61}$ Follow-up and treatment strategies for metastatic NSGCT are highly individualized, depending on the initial IGCCCG classification, prognostic factors, extent of disease, and response to initial chemotherapy. Patients with advanced/metastatic NSGCT should be referred to comprehensive cancer centers experienced in the management of this disease, and offered clinical trials.

\section{Seminoma}

\section{Clinical Stage I Seminoma: Surveillance}

The risk of relapse after orchiectomy for clinical stage I seminoma is approximately $15 \%$ to $20 \%$. A 2004 modeling study suggested that tumors $4 \mathrm{~cm}$ or greater or those with rete testis invasion were more likely to relapse. ${ }^{62}$ However, the significance of these 2 variables could not be validated in a larger cohort on multivariable analysis; on univariable analysis, and for the second study, only tumor size, treated as a continuous variable, was a significant predictor for recurrence. ${ }^{63}$ Although some investigators use a risk-adapted model for their management of clinical stage I seminoma and offer adjuvant treatment for their patients with larger tumors and rete testis invasion, ${ }^{64}$ most consensus-based groups recommend active surveillance, with treatment reserved exclusively for tumors that relapse, irrespective of tumor size or rete testis invasion.

In the past, the NCCN Guidelines recommended the intensive schedule of CT imaging originally described by the investigators at Princess Margaret Hospital, which consisted of 18 CT scans over 10 years. ${ }^{65}$ Using this approach, 15\% of 453 patients relapsed, $14 \%$ with stage IIC disease higher (Chung P, personal communication, 2011). Survival approached 100\% for those who experienced relapse because of the availability of excellent salvage regimens, such as radiotherapy for patients with smaller tumors and cisplatin-based chemotherapy for those with bulkier disease or distant relapse. Concerns about compliance, cost, and exposure to ionizing radiation led other investigators to select less-intense imaging schedules. In a study of 394 prospectively studied Danish patients who underwent 5 CT scans over 5 years, $17 \%$ relapsed, $26 \%$ with stage IIC disease or higher. ${ }^{19}$ Again, no testicular cancer-re- lated deaths were seen because of the efficacy of salvage therapy. The Spanish Germ Cell Cancer Group reported the results of their 8-CT scan protocol; there was a $16 \%$ relapse rate with no deaths secondary to testicular cancer. ${ }^{64}$ Given these reassuring data and also the fact that Canadian data from NSGCTs suggest that patient compliance is highest when imaging protocols are less intense, ${ }^{10}$ the authors recommended 6 or 7 CT scans for the first 5 years of follow-up rather than the 13 initially suggested by the NCCN Guidelines. ${ }^{65}$

The NCCN Guidelines still call for the use of CT scans rather than MRI despite concerns about the exposure to ionizing radiation from CT scans. ${ }^{8}$ MRI of the abdomen requires special imaging sequences and breath-holding techniques, and is not yet validated for testicular cancer surveillance across the nation but may be used in centers of excellence or under special circumstances on an individualized basis. The utility of MRI is being evaluated in a British prospective randomized trial evaluating 7 versus 2 CT or MRI scans over 5 years.

A final change in the current NCCN Guidelines for patients with clinical stage I seminoma is that tumor markers are now optional. ${ }^{8} \beta-\mathrm{HCG}$ and $\mathrm{LDH}$ are infrequently elevated at presentation and $\mathrm{t}$ relapse in patients with seminoma. A recent Canadian study of 527 surveyed patients revealed that of the 64 patients (14\%) who experienced relapse, only 1 had an elevated tumor marker (LDH) before relapse was detected by other means. ${ }^{7}$ In no cases was treatment management defined or altered based solely on elevated tumor markers. These data suggest that tumor markers may be safely eliminated from the surveillance protocols for stage I seminoma. No surveillance series reports first relapse occurring in the lungs in patients with clinical stage I seminoma; therefore, routine chest imaging is now completely optional for patients without thoracic symptoms. NCCN Guidelines for surveillance for clinical stage I seminoma are presented in Table $7 .^{8}$

\section{Clinical Stage I Seminoma: Adjuvant Radiotherapy and Adjuvant Carboplatin}

Although modern radiotherapy is associated with fewer risks of second malignant neoplasms than were reported in older studies, ${ }^{66} \mathrm{NCCN}$ does not recommend the routine use of adjuvant therapy for patients with stage I seminoma. ${ }^{8}$ For the rare patient who receives radiotherapy, randomized data suggest that 20 Gy given to a small para-aortic (PA) strip field is 
Follow-Up of Patients With Testicular Cancer

Table 7 Clinical Stage I Seminoma: Surveillance After Orchiectomy

\begin{tabular}{llllll|} 
& \multicolumn{5}{c}{ Year (at month intervals) } \\
\cline { 2 - 6 } & $\mathbf{1}$ & $\mathbf{2}$ & $\mathbf{3}$ & $\mathbf{4}$ & $\mathbf{5}$ \\
\hline H\&Pa,b & Every 3-6 mo & Every 6-12 mo & Every 6-12 mo & Annually & Annually \\
Abdominal/ & At 3, 6, & Every 6-12 mo & Every 6-12 mo & Every 12-24 mo \\
pelvic CT & and 12 mo & & & \\
Chest x-ray & & As clinically indicated, consider chest CT in symptomatic patients \\
\hline
\end{tabular}

${ }_{b}^{a}$ Serum tumor markers are optional.

Testicular ultrasound for any equivocal exam.

From the NCCN Clinical Practice Guidelines in Oncology: Testicular Cancer, Version 2, 2015. Available at: NCCN.org. Accessed May 28, 2015; with permission. To view the most recent version of these guidelines, visit NCCN.org.

just as effective as the use of higher doses and larger "dog-leg" fields based on the results of 2 prospective randomized trials. ${ }^{67,68}$ In the TE10 trial, 478 patients were randomized to either the smaller PA strip fields or the larger, more traditional dog-leg fields. Although the PA strip fields were associated with more pelvic relapses than the traditional dog-leg fields, the overall risk of relapse for either field arrangement was only $4 \%{ }^{68}$ The reason for the higher percentage of systemic relapses in the dog-leg field group is unclear. In a second study, TE18, 625 patients were randomized to either 20 or 30 Gy of radiotherapy given by either PA strip or dog-leg fields as per the treating clinician's preference. ${ }^{69}$ With a median follow-up period of 7 years, 44 patients had experienced relapse, 43 of whom did so within 36 months of trial entry. Cause-specific survival was $95.1 \%$ for the $30-$ Gy arm and $96.8 \%$ for the 20 -Gy arm $(P=.83)$.

Carboplatin has also been subjected to phase III trials in this patient population. TE19 randomized 1467 patients between radiotherapy $(n=904)$ or a single course of carboplatin $(n=573)$. Of the 66 patients who experienced disease relapse, 63 did so within 3 years. With a median survival of 6.5 years, the relapse-free survival rate was $96.0 \%$ after radiotherapy and $94.7 \%$ after carboplatin. ${ }^{67}$ Only 1 patient experienced relapse beyond 36 months, suggesting that both imaging and intense clinical follow-up are unnecessary beyond 3 years. ${ }^{67}$

Guidelines for follow-up after adjuvant radiotherapy were selected to reflect the findings of the Medical Research Center (MRC)/EORT) randomized trials in clinical stage I seminoma (TE10, TE18, and TE19)..$^{50,70}$ In these trials, $58.1 \%$ of relapses in patients treated with dogleg radiotherapy could be identified based on physical examination and clinical symptoms; only $22.6 \%$ were identified based solely on a CT scan. In these studies of 1477 patients with clinical stage I seminoma randomized to radiotherapy or carboplatin chemotherapy, only $0.2 \%$ experienced relapse after 36 months; therefore, in the NCCN Guidelines, imaging after 36 months is optional. ${ }^{8}$ Although relapse patterns are slightly different for each treatment, the recommendations for follow-up are designed to simplify this phase of management, given the low likelihood of relapse after adjuvant therapy for clinical stage I seminoma (see Table 8$).{ }^{8}$

\section{Clinical Stage IIA and IIB Seminoma: Radiotherapy}

Stage II pure seminoma is rare and therefore no randomized protocols address treatment techniques or follow-up protocols. The single-institutional published risk of relapse after radiotherapy for clinical stage IIA and IIB seminoma is lower in modern series ${ }^{71}$ than was reported in older series, ${ }^{72}$ which tended to include patients treated over several decades. For patients with stage II disease, radiotherapy is directed to the retroperitoneum and upper pelvic nodes. $\mathrm{Pa}$ tients with stage IIA disease have a risk of treatment failure of $5 \%$ to $10 \%$, whereas this risk can be up to $10 \%$ to $15 \%$ for patients with stage IIB disease. ${ }^{73}$

In one prospective German study evaluating slightly smaller radiotherapy fields than those traditionally used, 94 patients with stage II disease were recruited from 30 centers, ${ }^{74}$ with 87 available for analysis. Of these, 4 experienced relapse: 2 with stage IIA disease and 2 with stage IIB disease. Among these patients, 2 had mediastinal and 1 had pulmonary and mediastinal disease at relapse. The final patient experienced relapse in the lateral aspect of the retroperitoneum. All patients were salvaged with cisplatin-based chemotherapy. The times to relapse in the study were longer than expected, 


\begin{tabular}{|c|c|c|c|c|c|}
\hline & \multicolumn{5}{|c|}{ Year (at month intervals) } \\
\hline & 1 & 2 & 3 & 4 & 5 \\
\hline$H \& P^{a, b}$ & Every 6-12 mo & Every 6-12 mo & Annually & Annually & Annually \\
\hline Abdominal/pelvic CT & Annually & Annually & Annually & - & - \\
\hline Chest x-ray & \multicolumn{5}{|c|}{ As clinically indicated, consider chest $\mathrm{CT}$ in symptomatic patients } \\
\hline
\end{tabular}

${ }_{b}^{a}$ Serum tumor markers are optional.

Testicular ultrasound for any equivocal exam.

From the NCCN Clinical Practice Guidelines in Oncology: Testicular Cancer, Version 2, 2015. Available at: NCCN.org. Accessed May 28, 2015; with permission. To view the most recent version of these guidelines, visit NCCN.org.

occurring at 10, 17, 33, and 40 months. Given the rarity of abdominal relapse and the late thoracic relapse, the guidelines have decreased the number of abdominopelvic CT scans needed over time. Chest radiographs are obtained episodically, but clinicians are encouraged to obtain a chest CT for any patient with thoracic symptoms. NCCN Guidelines for follow-up after radiotherapy for stage II seminoma are presented in Table 9.8

\section{Clinical Stage II and III Seminoma: Chemotherapy With or Without Surgery}

The Spanish Germ Cell Cancer Group reported on a series of 18 patients with clinical stage IIA seminoma who received 3 cycles of BEP or 4 cycles of EP chemotherapy with no reported failures. ${ }^{75}$ Given the small number of patients studied, no separate guidelines were developed for these patients. Patients with clinical stage IIB, IIC, and III disease have higher rates of failure than those with IIA and are followed differently. Patients whose tumor markers are negative and have either no residual mass on imaging or a residual mass measuring less than $3 \mathrm{~cm}$ are unlikely to experience treatment failure and may be followed. ${ }^{76,77}$ Early data from Memorial Sloan Kettering Cancer Center revealed that RPLND in 28 patients with masses less than $3 \mathrm{~cm}$ yielded only necrotic material with no subsequent failures. ${ }^{77} \mathrm{NCCN}$ Guidelines for these patients are presented in Table $10 .{ }^{8}$ By comparison, $30 \%$ of 27 patients with residual masses $3 \mathrm{~cm}$ or greater had residual active cancer in their RPLND specimens. Similar data come from a 78-patient study reported by Flechon et al. ${ }^{78}$ Only patients with residual masses greater than 3 $\mathrm{cm}$ had viable tumor in their resected specimens (13\%). In that study, patients who had viable resected tumor or elevated tumor markers experienced treatment failure very quickly, within a month or two. However, some patients with residual masses, including masses greater than $3 \mathrm{~cm}$, who were followed experienced tumor shrinkage for up to 12 months, suggesting that the residual masses seen on CT scans after chemotherapy for seminoma can take a year or more to shrink. For those with a residual mass measuring $3 \mathrm{~cm}$ or greater, either serial CTs starting 3 months after treatment or a PET/ CT scan at 6 weeks may be undertaken with biopsy, and then salvage therapy as needed for those with residual cancer. ${ }^{76,77}$ Again, patients needing salvage therapy should be managed at referral centers with expertise in

\begin{tabular}{|lllllc|}
\hline Table 9 Clinical Stage IIA and Non-Bulky IIB Seminoma: Surveillance After \\
Radiotherapy
\end{tabular}

aSerum tumor markers optional.

bTesticular ultrasound for any equivocal exam.

'Chest x-ray may be used for routine follow-up, but chest CT is preferred in the presence of thoracic symptoms.

From the NCCN Clinical Practice Guidelines in Oncology: Testicular Cancer, Version 2, 2015. Available at: NCCN.org. Accessed May 28, 2015; with permission. To view the most recent version of these guidelines, visit NCCN.org. 


\begin{tabular}{|c|c|c|c|c|c|}
\hline & \multicolumn{5}{|c|}{ Year (at month intervals) } \\
\hline & 1 & 2 & 3 & 4 & 5 \\
\hline \multirow{2}{*}{$\begin{array}{l}\mathrm{H} \& \mathrm{P} \text { and markers } \\
\text { Abdominal/pelvic } \mathrm{CT}^{\mathrm{b}}\end{array}$} & Every 2 mo & Every 3 mo & Every $6 \mathrm{mo}$ & Every $6 \mathrm{mo}$ & Annually \\
\hline & \multicolumn{5}{|c|}{$\begin{array}{l}\text { Abdominal/pelvic CT at 3-6 months, then as clinically indicated } \\
\text { PET scan as clinically indicated }\end{array}$} \\
\hline Chest x-rayc & Every $2 \mathrm{mo}^{\mathrm{d}}$ & Every $3 \mathrm{mo}^{\mathrm{d}}$ & Annually & Annually & Annually \\
\hline
\end{tabular}

aTesticular ultrasound for any equivocal exam.

bPatients with PET-negative residual mass measuring $>3 \mathrm{~cm}$ following chemotherapy should undergo an abdominopelvic CT scan every 6 months for the first year then annually for five years.

'Chest x-ray may be used for routine follow-up but chest CT is preferred in the presence of thoracic symptoms.

${ }^{\mathrm{d} A d d}$ chest $\mathrm{CT}$ if supradiaphragmatic disease present at diagnosis.

From the NCCN Clinical Practice Guidelines in Oncology: Testicular Cancer, Version 2, 2015. Available at: NCCN.org. Accessed May 28, 2015; with

permission. To view the most recent version of these guidelines, visit NCCN.org.

germ cell tumors and, because of the specialized nature of follow-up in these patients, standardized guidelines for follow-up cannot be provided.

\section{Conclusions}

The goal of follow-up surveillance is to identify relapse early enough that effective salvage therapy can be given. The available literature was used to identify the risk of relapse by tumor type and stage, taking into account the absolute risk of recurrence, the modality most likely to identify recurrence, and the time frame over which recurrence was most likely to occur. These considerations were used to inform this article on follow-up after treatment for testicular cancer.

\section{References}

1. Nigam $M$, Aschebrook-Kilfoy $B$, Shikanov $S$, Eggener S. Increasing incidence of testicular cancer in the United States and Europe World J Urol 2015;33:623631.

2. Rosen A, Jayram G, Drazer M, Eggener SE. Global trends in testicular cancer incidence and mortality. Eur Urol 2011;60:374-379.

3. Power DA, Brown RS, Brock CS, et al. Trends in testicular carcinoma in England and Wales, 1971-99. BJU Int 2001;87:361-365.

4. Daugaard G, Gundgaard MG, Mortensen MS, et al. Surveillance for stage I nonseminoma testicular cancer: outcomes and long-term follow-up in a population-based cohort. J Clin Oncol 2014;32:3817-3823.

5. Kollmannsberger C, Tandstand T, Bedard PL, et al. Patterns of relapse in patients with clinical stage I testicular cancer managed with active surveillance. J Clin Oncol 2015;33:51-57.

6. Groll RJ, Warde P, Jewett MA. A comprehensive systematic review of testicular germ cell tumor surveillance. Crit Rev Oncol Hematol 2007;64:182-197.

7. Vesprini D, Chung $P$, Tolan $S$, et al. Utility of serum tumor markers during surveillance for stage I seminoma. Cancer 2012;118:5245-5250.

8. Motzer RJ, Jonasch E, Agarwal N, et al. NCCN Clinical Practice Guidelines in Oncology: Testicular Cancer. Version 2, 2015. Available at: NCCN.org. Accessed May 27, 2015.

9. Yu HY, Madison RA, Setodji CM, Saigal CS. Quality of surveillance for stage I testis cancer in the community. J Clin Oncol 2009;27:4327-4332.

10. Ernst DS, Brasher P, Venner PM, et al. Compliance and outcome of patients with stage 1 non-seminomatous germ cell tumors (NSGCT) managed with surveillance programs in seven Canadian centres. Can J Urol 2005;12(2): 2575-80.

11. Freedman LS, Jones WG, Peckham MJ, et al. Histopathology in the prediction of relapse of patients with stage i testicular teratoma treated by orchidectomy alone. Lancet 1987;330:294-298

12. Ondrus D, Matoska J, Belan V, et al. Prognostic factors in clinical stage I nonseminomatous germ cell testicular tumors: rationale for different riskadapted treatment. Eur Urol 1998;33:562-566.

13. Freedman LS, Parkinson MC, Jones WG, et al. Histopathology in the prediction of relapse of patients with stage I testicular teratoma treated by orchidectomy alone. Lancet 1987;2:294-298.

14. Albers $P$, Siener $R$, Kliesch $S$, et al. Risk factors for relapse in clinical stage $I$ nonseminomatous testicular germ cell tumors: results of the German Testicular Cancer Study Group Trial. J Clin Oncol 2003;21:1505-1512.

15. Tandstad T, Dahl O, Cohn-Cedermark G, et al. Risk-adapted treatment in clinical stage I nonseminomatous germ cell testicular cancer: the SWENOTECA management program. J Clin Oncol 2009;27 2122-2128.

16. Sturgeon JF, Moore MJ, Kakiashvili DM, et al. Non-risk-adapted surveillance in clinical stage I nonseminomatous germ cell tumors: the Princess Margaret Hospital's experience. Eur Urol 2011;59:556-562.

17. Kollmannsberger $C$, Tyldesley $S$, Moore $C$, et al. Evolution in management of testicular seminoma: population-based outcomes with selective utilization of active therapies. Ann Oncol 2011;22:808-814.

18. Rustin GJ, Mead GM, Stenning SP, et al. Randomized trial of two or five computed tomography scans in the surveillance of patients with stage I nonseminomatous germ cell tumors of the testis: Medical Research Council Trial TE08, ISRCTN56475197-the National Cancer Research Institute Testis Cancer Clinical Studies Group. J Clin Oncol 2007;25:1310-1315.

19. Daugaard G, Petersen PM, Rorth M. Surveillance in stage I testicular cancer. APMIS 2003;111:76-83; discussion 83-75.

20. Read G, Stenning SP, Cullen MH, et al. Medical Research Council prospective study of surveillance for stage I testicular teratoma. Medical Research Council Testicular Tumors Working Party. J Clin Oncol 1992;10:1762-1768.

21. Colls BM, Harvey VJ, Skelton L, et al. Late results of surveillance of clinical stage I nonseminoma germ cell testicular tumours: 17 years' experience in a national study in New Zealand. BJU Int 1999;83:76-82.

22. Cathomas R, Hartmann M, Krege S, et al. Interdisciplinary evidence-based recommendations for the follow-up of testicular germ cell cancer patients. Onkologie 2011;34:59-64.

23. Souchon R, Hartmann $M$, Krege $S$, et al. Interdisciplinary evidence-based recommendations for the follow-up of early stage seminomatous testicular germ cell cancer patients. Strahlenther Onkol 2011;187:158-166.

24. Schmoll $\mathrm{HJ}$, Jordan $\mathrm{K}$, Huddart $\mathrm{R}$, et al. Testicular non-seminoma: ESMO Clinical Practice Guidelines for diagnosis, treatment and follow-up. Ann Oncol 2010;21(Suppl 5):v147-154.

25. van As NJ, Gilbert DC, Money-Kyrle J, et al. Evidence-based pragmatic guidelines for the follow-up of testicular cancer: optimising the detection of relapse. Br J Cancer 2008;98:1894-1902.

26. Vergouwe Y, Steyerberg EW, Eijkemans MJ, et al. Predictors of occult metastasis in clinical stage I nonseminoma: a systematic review. J Clin Oncol 2003;21:4092-4099.

27. Bokemeyer C, Kuczyk MA, Serth J, et al. Treatment of clinical stage I testicular cancer and a possible role for new biological prognostic parameters. J Cancer Res Clin Oncol 1996;122:575-584. 
28. Divrik RT, Akdogan B, Ozen H, Zorlu F. Outcomes of surveillance protocol of clinical stage I nonseminomatous germ cell tumors-is shift to risk adapted policy justified? J Urol 2006;176:1424-1429; discussion 1429-1430.

29. Sogani PC, Perrotti M, Herr HW, et al. Clinical stage I testis cancer: long-term outcome of patients on surveillance. J Urol 1998;159:855-858.

30. Maroto P, Garcia del Muro X, Aparicio J, et al. Multicentre risk-adapted management for stage I non-seminomatous germ cell tumours. Ann Oncol 2005;16:1915-1920.

31. Albers $P$, Siener $R$, Krege $S$, et al. Randomized phase III trial comparing retroperitoneal lymph node dissection with one course of bleomycin and etoposide plus cisplatin chemotherapy in the adjuvant treatment of clinical stage I Nonseminomatous testicular germ cell tumors: AUO trial AH 01/94 by the German Testicular Cancer Study Group. J Clin Oncol 2008;26:2966-2972.

32. Kollmannsberger $\mathrm{C}$, Moore $\mathrm{C}$, Chi $\mathrm{KN}$, et al. Non-risk-adapted surveillance for patients with stage I nonseminomatous testicular germ-cell tumors: diminishing treatment-related morbidity while maintaining efficacy. Ann Oncol 2010;21:1296-1301.

33. Steiner H, Zangerl F, Stohr B, et al. Results of bilateral nerve sparing laparoscopic retroperitoneal lymph node dissection for testicular cancer. J Urol 2008;180:1348-1352; discussion 1352-1343.

34. Richie JP. Clinical stage 1 testicular cancer: the role of modified retroperitoneal lymphadenectomy. J Urol 1990;144:1160-1163.

35. Weissbach L, Bussar-Maatz R, Flechtner H, et al. RPLND or primary chemotherapy in clinical stage IIA/B nonseminomatous germ cell tumors? Results of a prospective multicenter trial including quality of life assessment. Eur Urol 2000;37:582-594.

36. 36. Stephenson AJ, Bosl GJ, Motzer RJ, et al. Nonrandomized comparison of primary chemotherapy and retroperitoneal lymph node dissection for clinical stage IIA and IIB nonseminomatous germ cell testicular cancer. J Clin Oncol 2007;25:5597-5602.

37. Donohue JP, Thornhill JA, Foster RS, et al. The role of retroperitoneal lymphadenectomy in clinical stage B testis cancer: the Indiana University experience (1965 to 1989). J Urol 1995;153:85-89.

38. Rabbani F, Sheinfeld J, Farivar-Mohseni H, et al. Low-volume nodal metastases detected at retroperitoneal lymphadenectomy for testicular cancer: pattern and prognostic factors for relapse. J Clin Oncol 2001;19:2020-2025

39. Williams SD, Stablein DM, Einhorn LH, et al. Immediate adjuvant chemotherapy versus observation with treatment at relapse in pathological stage II testicular cancer. N Engl J Med 1987;317:1433-1438.

40. Horwich A, Stenning S. Initial chemotherapy for stage II testicular nonseminoma. World J Urol 1994;12:148-150.

41. Peckham MJ, Hendry WF. Clinical stage II non-seminomatous germ cell testicular tumours. Results of management by primary chemotherapy. Br J Urol 1985;57:763-768

42. Vogelzang NJ, Fraley EE, Lange PH, et al. Stage II nonseminomatous testicular cancer: a 10-year experience. J Clin Oncol 1983;1:171-178.

43. Motzer RJ, Sheinfeld J, Mazumdar M, et al. Etoposide and cisplatin adjuvant therapy for patients with pathologic stage II germ cell tumors. J Clin Oncol 1995;13:2700-2704.

44. Grimison PS, Stockler MR, Thomson DB, et al. Comparison of two standard chemotherapy regimens for good-prognosis germ cell tumors: updated analysis of a randomized trial. J Natl Cancer Inst 2010;102:1253-1262.

45. Logothetis CJ, Swanson DA, Dexeus F, et al. Primary chemotherapy for clinical stage II nonseminomatous germ cell tumors of the testis: a follow-up of 50 patients. J Clin Oncol 1987;5:906-911.

46. Krege S, Beyer J, Souchon R, et al. European consensus conference on diagnosis and treatment of germ cell cancer: a report of the second meeting of the European Germ Cell Cancer Consensus Group (EGCCCG): part II. Eur Urol 2008;53:497-513.

47. Ehrlich Y, Brames MJ, Beck SD, et al. Long-term follow-up of Cisplatin combination chemotherapy in patients with disseminated nonseminomatous germ cell tumors: is a postchemotherapy retroperitoneal lymph node dissection needed after complete remission? J Clin Oncol 2010;28:531-536.

48. Beck SD, Foster RS. Management of the post chemotherapy subcentimeter residual mass: the case for observation. World J Urol 2009;27:485-488.

49. Panicek DM, Toner GC, Heelan RT, Bosl GJ. Nonseminomatous germ cell tumors: enlarging masses despite chemotherapy. Radiology 1990;175:499-502.

50. Fossa SD, Aass N, Ous S, et al. Histology of tumor residuals following chemotherapy in patients with advanced nonseminomatous testicular cancer. J Urol 1989;142:1239-1242.

51. Xiao H, Sheinfeld J, Motzer RJ. Adjuvant chemotherapy for testicular cancer. Surg Oncol Clin N Am 1997;6:863-878.

52. Kuczyk M, Machtens S, Stief C, Jonas U. Management of the postchemotherapy residual mass in patients with advanced stage non-seminomatous germ cell tumors (NSGCT). Int J Cancer 1999;83:852-855.

53. Toner GC, Panicek DM, Heelan RT, et al. Adjunctive surgery after chemotherapy for nonseminomatous germ cell tumors: recommendations for patient selection. J Clin Oncol 1990;8:1683-1694.

54. Baniel J, Foster RS, Gonin R, et al. Late relapse of testicular cancer. J Clin Oncol 1995;13:1170-1176.
55. Albers P, Ganz A, Hannig E, et al. Salvage surgery of chemorefractory germ cell tumors with elevated tumor markers. J Urol 2000;164:381-384.

56. Geldart TR, Gale J, McKendrick J, et al. Late relapse of metastatic testicular nonseminomatous germ cell cancer: surgery is needed for cure. BJU Int 2006;98:353-358.

57. Gietema JA, Meinardi MT, Sleijfer DT, et al. Routine chest X-rays have no additional value in the detection of relapse during routine follow-up of patients treated with chemotherapy for disseminated non-seminomatous testicular cancer. Ann Oncol 2002;13:1616-1620.

58. Fossa SD, Stenning SP, Gerl A, et al. Prognostic factors in patients progressing after cisplatin-based chemotherapy for malignant non-seminomatous germ cell tumours. Br J Cancer 1999;80:1392-1399.

59. Lorch A, Beyer J, Bascoul-Mollevi C, et al. Prognostic factors in patients with metastatic germ cell tumors who experienced treatment failure with cisplatinbased first-line chemotherapy. J Clin Oncol 2010;28:4906-4911.

60. Einhorn LH, Williams SD, Chamness A, et al. High-dose chemotherapy and stem-cell rescue for metastatic germ-cell tumors. N Engl J Med 2007;357:340348.

61. Motzer RJ, Geller NL, Tan CC, et al. Salvage chemotherapy for patients with germ cell tumors. The Memorial Sloan-Kettering Cancer Center experience (1979-1989). Cancer 1991;67:1305-1310.

62. Warde $\mathrm{P}$, Specht L, Horwich A, et al. Prognostic factors for relapse in stage I seminoma managed by surveillance: a pooled analysis. J Clin Oncol 2002;20:4448-4452.

63. Chung PW, Daugaard G, Tyldesley S, et al. Prognostic factors for relapse in stage I seminoma managed with surveillance: a validation study [abstract]. J Clin Oncol 2010;28(Suppl):Abstract 4535.

64. Aparicio J, Maroto P, del Muro XG, et al. Risk-adapted treatment in clinical stage I testicular seminoma: the third Spanish Germ Cell Cancer Group study. J Clin Oncol 2011;29:4677-4681.

65. Motzer RJ, Agarwal N, Beard C, et al. NCCN Clinical Practice Guidelines in Oncology: Testicular Cancer. Version 1, 2011. To view the most recent version of these guidelines, visit NCCN.org.

66. Beard CJ, Travis LB, Chen MH, et al. Outcomes in stage I testicular seminoma: a population-based study of 9193 patients. Cancer 2013;119:2771-2777.

67. Oliver RT, Mead GM, Rustin GJ, et al. Randomized trial of carboplatin versus radiotherapy for stage I seminoma: mature results on relapse and contralateral testis cancer rates in MRC TE19/EORTC 30982 study (ISRCTN27163214). J Clin Oncol 2011;29:957-962.

68. Fossa SD, Horwich A, Russell JM, et al. Optimal planning target volume for stage I testicular seminoma: A Medical Research Council randomized trial. Medical Research Council Testicular Tumor Working Group. J Clin Oncol 1999; 17:1146

69. Mead GM, Fossa SD, Oliver RT, et al. Randomized trials in 2466 patients with stage I seminoma: patterns of relapse and follow-up. J Natl Cancer Inst 2011;103:241-249.

70. Horwich A, Sleijfer DT, Fossa SD, et al. Randomized trial of bleomycin, etoposide, and cisplatin compared with bleomycin, etoposide, and carboplatin in good-prognosis metastatic nonseminomatous germ cell cancer: a Multiinstitutional Medical Research Council/European Organization for Research and Treatment of Cancer Trial. J Clin Oncol 1997;15: 1844-1852.

71. Schmidberger $H$, Bamberg $M$, Meisner $C$, et al. Radiotherapy in stage IIA and IIB testicular seminoma with reduced portals: a prospective multicenter study. Int J Radiat Oncol Biol Phys 1997;39:321-326.

72. Classen J, Schmidberger H, Meisner C, et al. Radiotherapy for stages IIA/B testicular seminoma: final report of a prospective multicenter clinical trial. J Clin Oncol 2003;21:1101-1106.

73. Wilder RB, Buyyounouski MK, Efstathiou JA. Radiotherapy treatment planning for testicular seminoma. Int J Radiat Oncol Biop Phys 2012;83:445-452.

74. Classen J, Souchon R, Hehr T, Bamberg M. Radiotherapy for early stages testicular seminoma: patterns of care study in Germany. Radiother Oncol 2002;63:179-186.

75. Garcia-del-Muro X, Maroto P, Guma J, et al. Chemotherapy as an alternative to radiotherapy in the treatment of stage IIA and IIB testicular seminoma: a Spanish Germ Cell Cancer Group Study. J Clin Oncol 2008;26:5416-5421.

76. Warde P, Huddart R, Bolton D, et al. Management of localized seminoma, stage I-II: SIU/ICUD Consensus Meeting on Germ Cell Tumors (GCT), Shanghai 2009. Urology 2011;78:S435-443.

77. Herr HW, Sheinfeld J, Puc HS, et al. Surgery for a post-chemotherapy residual mass in seminoma. J Urol 1997;157:860-862.

78. Flechon A, Bompas E, Biron P, Droz JP. Management of post-chemotherapy residual masses in advanced seminoma. J Urol 2002;168:1975-1979. 